Ann. Biol. anim. Bioch. Biophys., 1979, 19 (3 A), 607-617.

\title{
Passage d'éléments d'origine microbienne à travers la muqueuse intestinale du lapin. Étude autoradiographique après marquage des microorganismes par la glycine tritiée
}

\author{
par M. PETITPREZ, R. MONCOULON, L. ALBERTINI, P. RAYNAUD \\ Laboratoire de Zoolechnie, E.N.S.A.T., \\ 145, avenue de Muret, 31076 Toulouse Cedex.
}

\begin{abstract}
Summary. Passage of bacterial material through the intestinal mucosa of rabbits. Autoradiographic study using tritiated glycine-labelled microorganisms.

Using autoradiography, the migration of bacterial material absorbed by the rabbit intestinal mucosa was studied in intestinal loops containing tritiated glycine-labelled microorganisms. These microorganisms adjoined the absorbing cells which took the bacterial glycine ; small lymphocytes carried if to the lamina propria. In that part of the villus, macrophages and lymphocytes conveyed the bacterial glycine in the blood and lymphatic vessels. In the spleen and mesenteric nodes, the lymphocytes, passed through the veinlet endothelium to migrate into the lymphatic vessels and lymphoid follicles. A part of the glycine was used for liver metabolism. The part unused by the organism, distributed over the whole small intestine by the circulation, was preferentially excreted by the jejunal mucosa goblet cells.
\end{abstract}

\section{Introduction.}

Il est admis depuis de nombreuses années que les mammifères vivent en symbiose avec la flore microbienne de leur tractus intestinal. Cette microflore effectue des synthèses protéiques, glucidiques, vitaminiques, etc... à partir de matériaux contenus dans le bol alimentaire ou excrétés par la muqueuse dans la lumière intestinale. L'organisme peut ensuite capter la plupart des composés ainsi élaborés. Si, dans cette association symbiotique, les synthèses bactériennes sont actuellement bien connues, il n'en est pas de même du comportement de la muqueuse vis-à-vis de la microflore, ni des mécanismes d'absorption de ses métabolites.

Les observations de Hampton ef Rosario (1965) en microscopie électronique ont révélé que des microorganismes intestinaux peuvent s'associer étroitement aux cellules épithéliales de la muqueuse. Par ailleurs, les cellules de Paneth douées de phagocyłose, renferment fréquemment des restes de microorganismes intestinaux (Balas, 1974). Les travaux de Grys (1966) prouvent que le cytoplasme des entérocytes contient 
du matériel bactérien ainsi que des fragments membranaires d'Escherichia coli. Enfin, dans une étude récente (Petitprez et al., 1978) nous avons pu montrer que le fer bactérien traverse l'épithélium intestinal, avant d'être pris en charge par des phagocytes mononucléés pour être acheminé vers le milieu intérieur. Ces différents travaux semblent indiquer que les constituants bactériens sont susceptibles de franchir la barrière intestinale pour passer ensuite dans la circulation.

Néanmoins, de nombreux points restent obscurs : en particulier demeurent indéterminées la façon dont s'effectuent les échanges entre bactéries et entérocytes, l'identité des cellules impliquées dans les diverses phases du transport ainsi que les voies d'acheminement des cellules mobiles. Pour tenter de lever ces indéterminations, nous avons choisi de marquer les microorganismes par la glycine tritiée, car, d'une part, la glycine est largement incorporée dans les métabolites bactériens, et, d'autre part, le tritium autorise l'élaboration d'histoautoradiogrammes de haute résolution. Les microorganismes ainsi marqués ont été mis au contact d'anses intestinales pendant des durées variables afin d'établir par analyse histoautoradiographique une cinétique du passage transmuqueux d'éléments de la microflore intestinale.

\section{Matériel et méthodes.}

Afin d'obtenir une vue dynamique précise du passage du matériel bactérien à travers la muqueuse intestinale, nous avons placé, pour des durées variables, des micro-organismes caecaux marqués par de la glycine tritiée, dans des anses intestinales isolées chez le Lapin.

\section{1. - Anses isolées.}

Nous avons réalisé, sur deux séries de 10 lapins de $2,5 \mathrm{~kg}$ en moyenne, des anses isolées de $15 \mathrm{~cm}$ environ, en utilisant la technique adoptée par Moncoulon et al. (1976). Pour une série les anses ont été isolées sur le duodénum pour l'autre série sur le jéjunum.

\section{2. - Obtention des microorganismes marqués.}

Les microorganismes sont isolés à partir de caecum de Lapin. Après abattage de l'animal, le contenu de l'organe est mis en suspension dans $300 \mathrm{ml} \mathrm{de} \mathrm{NaCl}$ à 9 p. 1000 , filtré sur gaze et centrifugé pendant $5 \mathrm{mn}$ à $2500 \mathrm{~g}$ afin d'éliminer les particules végétales. Le surnageant est repris, centrifugé à $18000 \mathrm{~g}$ pendant $20 \mathrm{mn}$, le culot ainsi obtenu renferme une grande partie des bactéries caecales. Un gramme de ce culot est mis en suspension dans $20 \mathrm{ml}$ du surnageant auxquels sont adjoints $20 \mathrm{mg}$ de peptone et $400 \mu \mathrm{Ci}$ de glycine ${ }^{3} \mathrm{H}$ (CEA Saclay). La culture ainsi réalisée est incubée $48 \mathrm{~h}$ à $38^{\circ} \mathrm{C}$.

Durant cette période des micro-prélèvements sont effectués à intervalles réguliers, permettant de réaliser des frottis que l'on soumet au procédé autoradiographique de Ficq (1961). Ceci nous a permis de vérifier que le marquage des microorganismes par la glycine augmente régulièrement jusqu'à la $6^{e}$ heure et atteint un maximum à partir de la $12^{\mathrm{e}}$ heure. Afin d'éliminer toute trace de glycine ${ }^{3} \mathrm{H}$ en solution, la suspension 
bactérienne est soumise à 3 lavages successifs à l'aide de liquide physiologique éliminé par centrifugation.

3. - Prélèvements et traitement des échantillons.

La suspension bactérienne est introduite par fractions de $2 \mathrm{ml}$ dans les anses intestinales préalablement lavées à l'aide d'une solution de $\mathrm{NaCl}$ à 9 p. 1000 à $38^{\circ} \mathrm{C}$. Les bactéries sont laissées au contact de la muqueuse intestinale pendant des durées variables : $30 \mathrm{mn}, 1 \mathrm{~h}, 2 \mathrm{~h}$ ou $4 \mathrm{~h}$.

Les Lapins sont ensuite sacrifiés. Le traitement histologique des échantillons d'organes prélevés est fait selon la méthode précédemment exposée (Petitprez et al., 1978) (coupes de $6 \mu \mathrm{m}$; émulsion nucléaire llford, Type K2 ; exposition : 21 jours). Chaque comptage du nombre de grains d'argent développé est rapporté à une aire de $100 \mu \mathrm{m}^{2}$ de structure cellulaire. Dans les figures 1 et 2 chaque moyenne est accompagnée de son erreur type. La comparaison des moyennes a été effectuée en utilisant le test « $\dagger$ » de Student.

\section{Résultats.}

\section{1. - Activité de l'anse isolée.}

Nous avons pu observer des bactéries associées à la muqueuse intestinale (photos 1 et 2). Elles sont accolées à un ou plusieurs entérocyłes et fréquemment englobées dans des coulées de mucus.

Au niveau de la jonction entre les microorganismes et les cellules épithéliales, la paroi bactérienne présente un éclaircissement qui s'accompagne fréquemment de modifications du cytoplasme bactérien suggérant une lyse progressive. Le marquage des microorganismes est parfois si étroitement associé aux entérocytes qu'il est difficile de préciser la position intra-entérocyłaire ou bien intra-bactérienne de la glycine tritiée.

Près des zones de jonction avec les microorganismes, l'épithélium muqueux est fréquemment infiltré par des mononucléaires assez volumineux qui sont vraisemblablement des plasmocytes.

Les entérocytes sont marqués très tôt (photo 3 ) (moins de $30 \mathrm{mn}$ après l'introduction des bactéries) dans leur cyłoplasme supra-nucléaire. L'activité de ces cellules augmente avec la durée de contact des microorganismes. Nous n'avons jamais observé de trace de radioactivité dans la partie infra-nucléaire des entérocytes; à ce niveau de la muqueuse, le marquage semble être exclusivement réparti dans les lymphocytes épithéliaux, situés dans les espaces de Grünhagen.

Ces petits lymphocytes sont intensément marqués dès la $30^{\mathrm{e}}$ minute (fig. 1 et 2 ). Le matériel bactérien franchit rapidement la barrière duodénale puisque en moins d'une heure, le marquage lymphocyłaire est maximal à ce niveau. Par contre dans les anses jéjunales, le marquage des petits lymphocytes n'augmente significativement qu'entre la $2^{\mathrm{e}}$ et la $4^{\mathrm{e}}$ heure. D'autre part, la quantité de glycine présente dans la muqueuse est moins importante dans les anses jéjunales que dans les anses duodénales. 


\section{PLANCHE}

Etude du passage de micro-organismes marqués par la glycine tritiée

à travers la muqueuse d'anses intestinales isolées

(Fixation : éthanol-acide acétique $3 / 1$; coloration : vert de méthyl-pyronine).

PHOTO 1. - Microorganisme étroitement accolé à la partie apicale des entérocytes (e). La paroi bactérienne présente, au contact des cellules épithéliales, un éclaircissement (flèche) qui s'accompagne d'une lyse du cytoplasme bactérien (Cy) (G: 3000 fois).

PHOTO 2. - Microorganisme intensément marqué par la glycine tritiée. Une extrémité de cette bactérie fait saillie à l'intérieur du cytoplasme d'un entérocyte (e). Dans cette zone, la paroi bactérienne n'est plus visible (flèche). Du marquage est passé dans le cytoplasme entérocyłaire (G: 2000 fois).

PHOTO 3. - Fragment de muqueuse d'une anse duodénale mise au contact de microorganismes marqués. La glycine bactérienne (flèche) est localisée dans la partie supra nucléaire des entérocytes (e). On note la présence de plusieurs petits lymphocytes (Ly) dont certains sont à proximité du matériel bactérien, alors que d'autres sont situés près de la Lamina propria (IP) (G:1500 fois). 

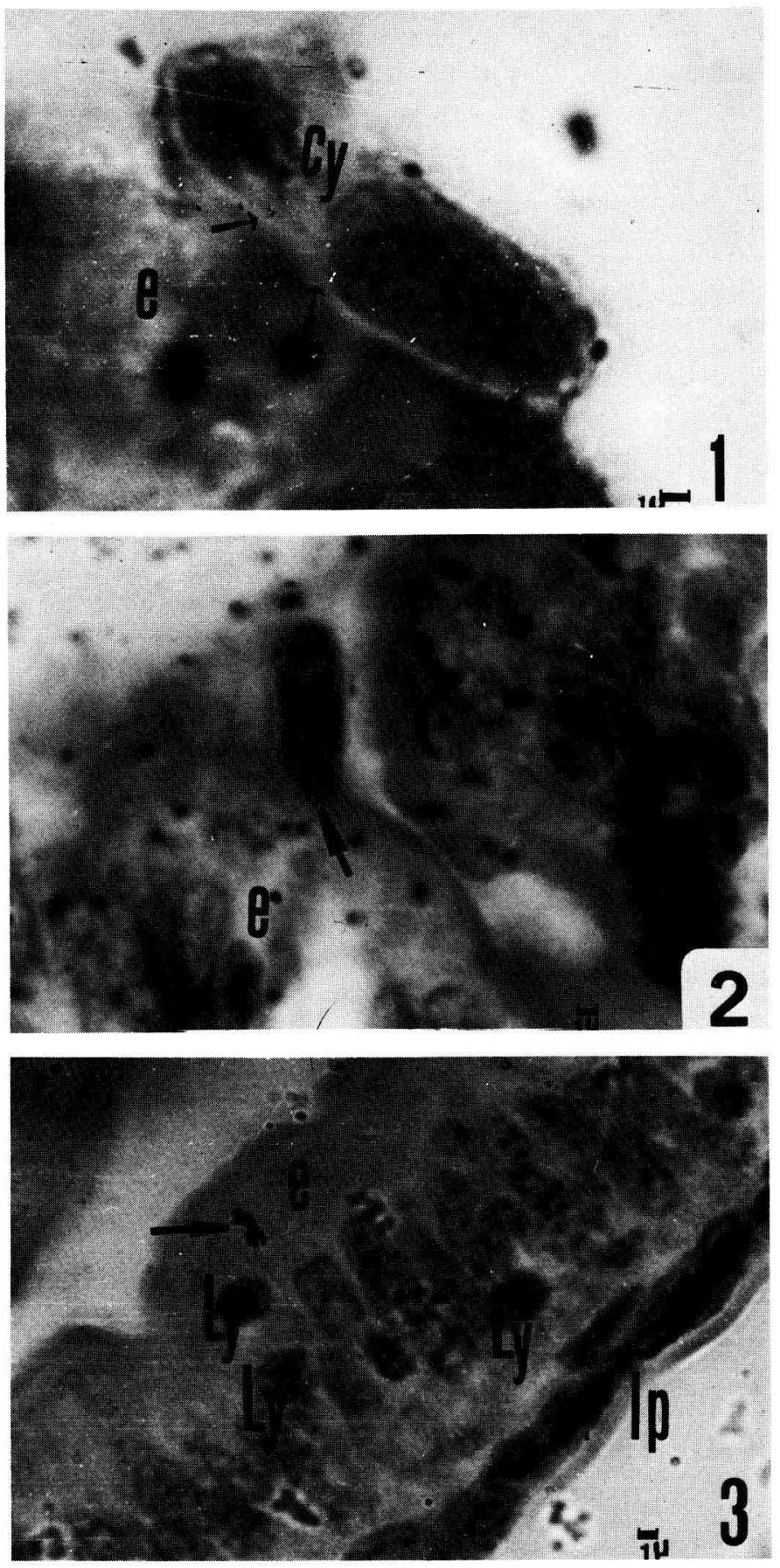
Dans la Lamina propria, l'activité est répartie sur deux types de cellules : des macrophages, cellules allongées situées essentiellement dans le tissu conjonctif, ef des grands lymphocytes, fréquents dans la partie vasculaire de l'axe de la villosité. Les grands lymphocytes des anses duodénales montrent un marquage maximal en moins de $2 \mathrm{~h}$.

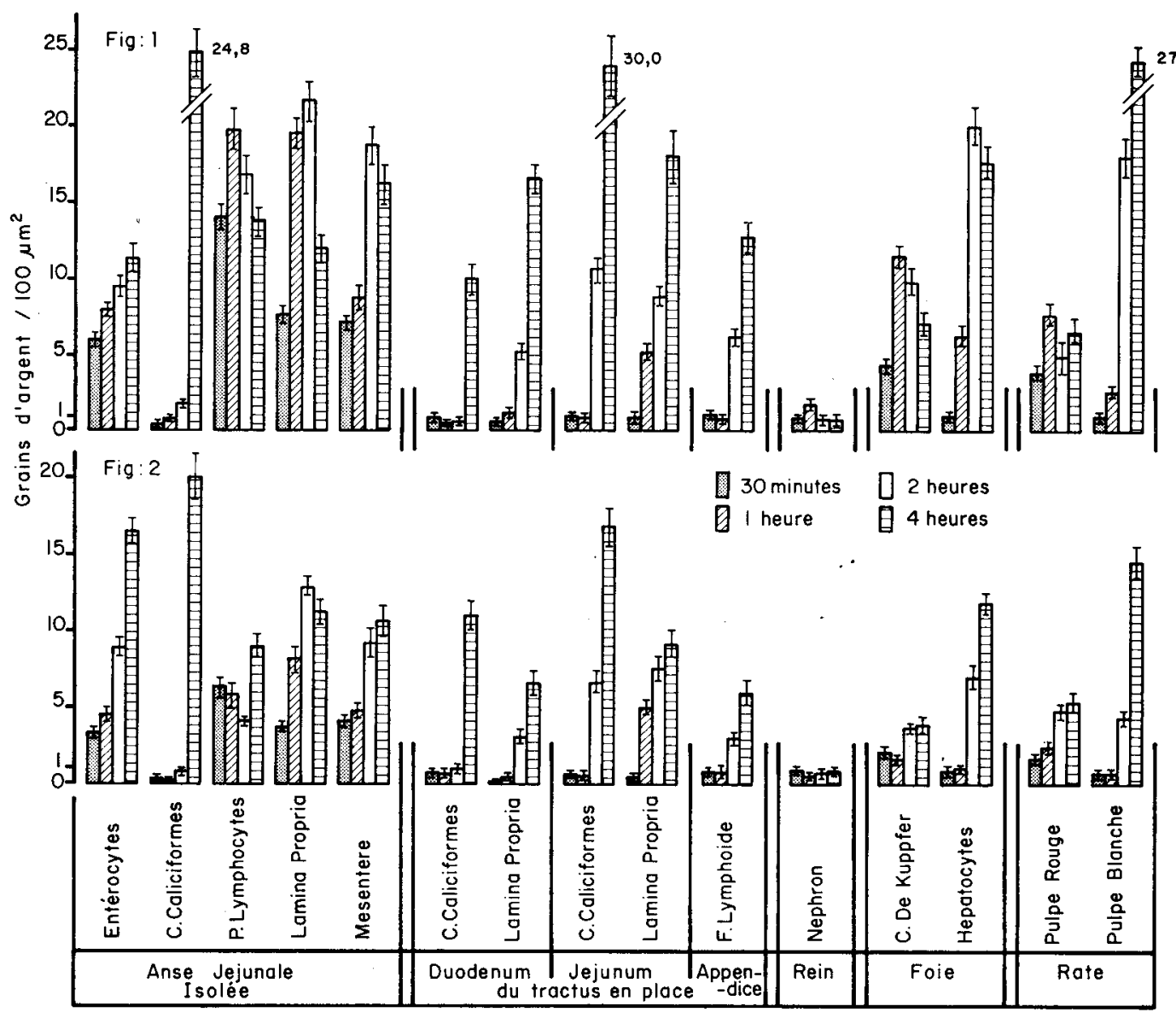

FIG. 1. - Activités tissulaires (exprimées en nombre de grains d'argent par $100 \mu \mathrm{m}^{2}$ ) d'organes prélevés chez des Lapins dont les anses duodénales isolées ont contenu une suspension de bactéries morquées par de la glycine tritiée $(20 \mu \mathrm{Ci} / \mathrm{ml})$, pendant des durées variables.

FIG. 2. - Activités tissulaires (exprimées en nombre de grains d'argent par $100 \mu \mathrm{m}^{2}$ ) d'organes prélevés chez des Lapins dont les anses jéjunales isolées ont contenu pendant des durées variables une suspension de bactéries marquées par de la glycine tritiée $(20 \mu \mathrm{mCi} / \mathrm{mi})$.

Dans le mésentère, le tritium est situé dans des lymphocytes. L'évolution du marquage de ces cellules est le reflet du marquage des lymphocytes de la Lamina propria. 
Les cellules caliciformes semblent avoir un comportement particulier au sein de la muqueuse. En effet, le marquage n'apparaît intensément qu'au cours de la 4e heure.

Dans les fragments de mésentère prélevés, nous avons pu observer des ganglions lymphatiques. Leur marquage débute toujours dans la zone centrale, au niveau des cordons folliculaires ( $2^{\mathrm{e}}$ heure) et se poursuit dans les follicules lymphoïdes (4e heure).

2. - Répartition de la glycine bactérienne dans le rein, le foie et la rate.

Le rein ne contient aucun marquage significatif ni dans les cellules du néphron, ni dans le tissu conjonctif.

Dans le foie, le marquage apparaît (après 1 h), dans les régions périvasculaires, le cytoplasme des cellules de Küppfer renfermant très tôt de la glycine bactérienne (fig. 1 et 2). Le marquage des hépatocytes ne se fait que plus tardivement (après $2 \mathrm{~h}$ ).

Dans la rate, l'activité débute dans la pulpe rouge, zone richement vascularisée, pour affecter ensuite les formations lymphoïdes de la pulpe blanche (fig. 1 et 2 ). Dans la pulpe rouge, les cellules marquées sont des macrophages ou des polymorphonucléaires. Par contre, dans la pulpe blanche, les molécules radioactives sont portées par des lymphocytes.

\section{3. - Activité des segments en place du tractus intestinal.}

Après introduction de bactéries dans les différentes anses isolées, tous les segments d'intestin grêle prélevés dans un délai de $30 \mathrm{mn}$ sonł dépourvus de radioactivité.

Une heure après l'introduction de bactéries dans l'anse isolée, les segments de duodénum n'ayant pas été en contact avec les bactéries marquées, sont dépourvus de radioactivité, alors que les segments jéjunaux sont marqués au niveau de la Lamina propria. Cette radioactivité du jéjunum augmente au cours de la $2^{e}$ heure et s'étend à l'ensemble de la muqueuse. D'autre part, après $4 \mathrm{~h}$ de contact, l'épithélium de fous les segments intestinaux renferme une radioactivité importante.

Nous avons noté un décalage entre la charge des segments duodénaux et jéjunaux; ces derniers sont plus intensément et plus rapidement marqués que les premiers.

D'autre part, de façon générale, le tractus intestinal des animaux porteurs d'une anse duodénale contient davantage de marquage que celui des Lapins munis d'une anse jéjunale.

Au sein de la muqueuse, ce sont presque exclusivement les cellules caliciformes qui portent le marquage. Cette activité est répartie à la périphérie des grains de mucus. Après libération des mucines dans la lumière intestinale le marquage des mucopolysaccharides est uniforme.

\section{Discussion.}

1. - Association des bactéries à la muqueuse intestinale.

De telles associations ont été mentionnées par quelques auteurs : Nelson et Mata (1970) ont trouvé des cocci accolés à la muqueuse de l'iléon, et Savage (1970) a observé des bâtonnets gram-positifs liés à l'apex des villosités. Des constatations similaires ont 
aussi été faites, en microscopie électronique, par Hampton et Rosario (1965) ; ces auteurs ont observé la formation de vésicules de pinocytose dans la zone de jonction entre microorganismes et cellules épithéliales. De ces travaux, il ressort que les entérocytes, lors de leur contact avec les microorganismes, entament simultanément une lyse et un processus d'endocytose du matériel bactérien.

Après pénétration dans l'entérocyłe, le matériel bactérien est vacuolisé dans des hétérophagolysosomes (Hampton et Rosario, 1965) au sein desquels s'effectue une digestion progressive aboutissant à la formation de figures myéliniques observées par Pitha (1968) et Balas (1974).

Les images que nous avons observées suggèrent une lyse progressive de la cellule bactérienne par les sécrétions de l'entérocyte, le matériel cytoplasmique bactérien pourrait être ensuite absorbé par cette même cellule épithéliale.

\section{2. - Rôle des lymphocytes épithéliaux.}

Les lymphocyłes épithéliaux sont les éléments les plus rapidement marqués dans la muqueuse. Ces lymphocytes sont toujours observés dans la couche épithéliale, au-dessous du niveau des noyaux des entérocytes. Ils ne le sont que très rarement vers la lumière intestinale. Ces petits lymphocytes ne peuvent donc pas assurer directement l'absorption de matériel bactérien. Ils pourraient plutôt permettre le transfert de ces éléments de l'entérocyte vers la Lamina propria.

L'épithélium intestinal renferme un grand nombre de petits lymphocytes. Des extractions onf été réalisées par Rudzik et Bienenstock (1974) sur la muqueuse de l'iléon. Ces auteurs trouvent que 90 p. 100 des cellules lymphoïdes de la muqueuse sont des lymphocytes, parmi lesquels la moitié environ est constituée de petits lymphocytes épithéliaux. Ces cellules principalement situées dans les espaces de Grünhagen, seraient capables d'émettre des pseudopodes et de pénétrer assez profondément dans les entérocytes adjacents. D'autre part, les lymphocytes qui arrivent près de la Lamina propria contiennent beaucoup plus de lysozomes et de vacuoles, que ceux situés à hauteur du noyau des entérocytes (Meader et Landers, 1967).

Ainsi les petits lymphocytes par leur grande motilité, seraient capables de capter le matériel bactérien contenu dans le cytoplasme des entérocytes et de le transférer, au sein de phagolysosomes, vers la Lamina propria.

\section{3. - Marquage des éléments cellulaires de la Lamina propria.}

Dans la Lamina propria, les cellules renfermant le matériel bactérien sont essentiellement des macrophages ef des grands lymphocytes. Nous n'avons que très rarement trouvé des petits lymphocytes. Le retard de marquage observé entre la Lamina propria ef l'épithélium suggère un délai de transfert du matériel bactérien.

La glycine tritiée des bactéries a donc été échangée au niveau de la membrane basale des entérocytes entre petits lymphocytes et macrophages. Certaines observations, réalisées en microscopie électronique, ont montré que la Lamina propria contient surtout des lymphocytes et des plasmocyłes (Kagnoff, 1974) associés à des macrophages (Clark, 1963). Ces rapports étroits entre les diverses cellules suggèrent un échange de matériel. En effet Deane (1964) a observé des vésicules identiques de part et d'autre de jonctions intercellulaires complexes. D'après cet auteur, les macro- 
phages, après avoir phagocyłé et dégradé les bactéries, transfèrent une partie de ce matériel aux plasmocyłes. Ces éléments constituent l'information nécessaire à la synthèse de gamma-globulines spécifiques (Deane, 1964).

Crabbe ef al. (1968) ont montré que le duodénum renferme trois fois plus de plasmocytes sécrétoires que l'iléon, que les animaux axéniques sécrètent de 10 à 15 fois moins d'IgA que les animaux conventionnels, et que la sécrétion d'anticorps par les cellules immuno-compétentes de l'intestin grêle se fait effectivement en réponse à une stimulation antigénique due à la flore bactérienne. Nous pouvons penser que cette plus grande activité sécrétoire du duodénum est à l'origine des absorptions plus rapides et plus importantes que nous avons observé dans cette partie de l'intestin grêle. Bien que Walker et Isselbacher (1974) aient pu montrer que l'absence d'IgA facilite l'absorption de certaines macromolécules, le rôle précis de la réaction immunitaire devra être étudié ultérieurement, car un problème subsiste au niveau de l'iléon. Riche en amas lymphoïdes et en plaques de Peyer, la partie distale de l'intestin grêle est celle qui sécrète le moins d'anticorps (Crabbe ef al., 1968) mais il semblerait que les cellules lymphoïdes ne soient jamais en contact avec les antigènes bactériens (Bienenstock et Dolezel, 1971), même si des microorganismes filamenteux peuvent être directement attachés à l'épithélium superficiel des plaques de Peyer (Abrams, 1977).

4. - Répartition de la glycine tritiée dans les ganglions lymphatiques le foie ef la rafe.

Les différents résultałs nous amènent à penser que la glycine tritiée, contenue dans les lymphocytes sanguins et mésentériques, pourrait être sélectivement orientée vers les formations lymphoïdes de l'organisme. Ce phénomène a d'ailleurs été déjà observé dans les ganglions lymphatiques (Gowans ef Knight, 1964).

La glycine bactérienne portée par les lymphocytes est distribuée vers les différents organes simultanément par la voie sanguine et la voie lymphatique.

Au niveau du foie, le matériel bactérien capté par les cellules de Küppfer serait ensuite transféré aux hépatocytes en vue d'une utilisation dans diverses synthèses.

\section{5. - Excrétion intestinale.}

Les résultats sont en accord avec les travaux de Petitprez ef al. (1978) montrant que l'excrétion du fer bactérien par la muqueuse intestinale se faif préférentiellement au niveau du jéjunum.

Le transport sanguin de la glycine bactérienne est assuré par des lymphocytes ef, dans la Lamina propria, elle est prise en charge par des macrophages. Les travaux de Mouriquand (1961), de Crosby (1963), de Cattan ef al. (1965) impliquent aussi les macrophages dans l'élimination intestinale du fer par sortie de ces cellules dans la lumière intestinale.

Nous avons trouvé dans l'épithélium muqueux, des cellules caliciformes très significativement marquées $4 \mathrm{~h}$ après la mise des bactéries au contact de l'anse isolée. Il pourrait donc exister, en plus de l'excrétion directe par les macrophages, un transfert de marquage sur les cellules caliciformes. 


\section{Conclusion.}

Les bactéries cæcales du Lapin, marquées in vitro au moyen de glycine ${ }^{3} \mathrm{H}$ ont été introduites dans des anses intestinales isolées chez le Lapin à l'abri de l'action lysante des sucs digestifs. Le devenir du marquage a pu être suivi avec précision, à l'échelle cellulaire, par histoautoradiographie de haute résolution, du fait de la faible énergie des particules émises.

Les entérocytes absorbent des éléments microbiens et leur font franchir la muqueuse intestinale. Le matériel bactérien issu des phagolysosomes est véhiculé par des cellules du système réticulo-endothélial : les phagocytes mononucléés qui emprunfent indifféremment les voies sanguine ou lymphatique. La glycine issue des corps bactériens a été retrouvée au niveau du foie et de la rate.

Chez le Lapin, les microorganismes cæcaux se développent sur un milieu constitué essentiellement de nutriments non absorbés ou libérés par l'intestin grêle. Par coprophagie, l'animal réingère une partie de ces bactéries qui, au niveau de l'intestin grêle, constituent un apport nutritif complémentaire. De ce faił les microorganismes du caecum jouent un rôle d'économie nutritionnelle par limitation des pertes.

Reçu en mars 1978.

Accepté en décembre 1978.

\section{Références}

ABRAMS G. D., 1977. Microbial effects on mucosal structure and function. Amer. J. clin. Nutr., 30, 1880-1886.

BALAS D., 1974. La cellule de Paneth. Etude histologique et histophysiologique. Thèse Doct. Etat, Toulouse. BIENENSTOCK Y. J., DOLEZEL J., 1971. Lack of antibody in Peyer's patches. Gastroenterology, 61, 940-941.

CATTAN O., DEBRAY C. H., JORI J. P., MARCHE C. P., 1965. Le macrophage péricapillaire du chorion villositaire duodénal : plaque tournante du métabolisme martial. Bull. Soc. Hématol., 6, 431-435.

CLARK S. L., 1963. Isolated environment of lymphoid tissues of the intestine. Fed. Proc., 22, 1339-1348.

CRABBE P. A., BAZIN H., EYSSEN H., HEREMANS H., 1968. The normal microbial flora as a major stimulus for proliferation of plasma cells synthesezing $\operatorname{lgA}$ in the gut. Inf. Arch. Allerg., 34, 362-375.

CROSBY W. H., 1963. The control of iron balance by the intestinal mucosa. Blood, 22, 441-449.

DEANE H. W., 1964. Some electron microscopic observations on the lamina propria of the gut. Anat. Rec., 149, 453-473.

FICQ A., 1961. Contribution à l'éfude du métabolisme cellulaire au moyen de la méthode autoradiographique. Monographie. Inst. interuniv. Sci. nuclé. Bruxelles.

GANTER P., MARCHE C. P., 1970. Histochimie des mucines gastro-intestinales de l'homme. Ann. Anat. Pathol., 15, 3, 321-346.

GOWANS J. L., KNIGHT E. J., 1964. The route of recirculation of lymphocytes in the rat. Proc. roy. Soc. Brit., 159, 257-282.

GRYS S., 1966. The mode of passage of $E$. coli and its antigens across the intestinal barrier of sheep. Intracellular localization of bacterial antigens in some internal organs. Polon. Archiv. Wet., 10, 243-240.

HAMPTON J. C., ROSARIO B., 1965. The attachment of microorganisms to epithelial cells in the distalileum of the mouse. Lab. Invest., 14, 1465-1481. 
KAGNOFF M. F., 1974. Induction and paralysis : a conceptual framework from which to examine the intestinal immune system. Gastroenterology, 66, 1240-1256.

LEVIN D. M., ROSENSTREICH D. L., REYNOLDS H. Y., 1973. Immunologic responses in the gastrointestinal tract of the guinea pig. I. - Characterization of Peyer's patch cells. J. Immunol., 111, 980-983.

MEADER R. D., LANDERS D. F., 1967. Electron and light microscopic observations on relationships between lymphocytes and intestinal epithelium. Amer. J. Anat., 121, 763-773.

MONCOULON R., MANDJOBA V., PETITPREZ M., 1976. Etude de l'excrétion intestinale du fer chez le Lapin par la méthode des anses isolées. 1er Congr. int. Cunic., Dijon, Communic. $n^{\circ} 60$.

MOURIQUAND Cl., 1961. Exploration des réserves de fer par des techniques histologiques. Path. Biol., 9, 1557-1560.

NELSON D. P., MATA J. L., 1970. Bacterial flora associated with human gastro-intestinal mucosa. Gastroenterology, 58, 56-66.

PETITPREZ M., MONCOULON R., ALBERTINI L., RAYNAUD P., 1978. Comportement de la muqueuse intestinale vis-à-vis d'entérobactéries par ${ }^{59} \mathrm{Fe}$. Etude autoradiographique chez le lapin. Ann. Biol. anim. Bioch. Biophys., 18, 1351-1361.

PITHA J., 1968. The fine structure of membraneous inclusions in the Paneth cells of rabbit. Z. Zellforsch., 90, 563-569.

RUDZIK O., BIENENSTOCK J., 1974. Isolation and characteristics of gut mucosal lymphocytes. Lab. Invest., 30, 260-266.

SAVAGE D. C., 1970. Associations of indigeneous microorganisms with gastro-intestinal mucosal epithelium. Amer. J. clin. Nutrit., 23, 1495-1501.

TONER P. G., 1968. Cytology of intestinal epithelial cells. Int. Rev. Cytol., 24, 232-343.

WALKER W. A., ISSELBACHER K. J., 1974. Uptake and transport of macromolecules by the infestine. Gastroenterology, 67, 3, 531-550.

WEISS L., 1964. The white pulp of the spleen relationships of arterial vessels reticulum and free cells in the periarterial lymphatic sheath. Bull. John Hopkins Hosp., 115, 99-173. 